\title{
Differences between searching among objects and searching among holes
}

\author{
JOHAN HULLEMAN and GLYN W. HUMPHREYS \\ University of Birmingham, Birmingham, England
}

\begin{abstract}
We report results from six experiments in which participants had to search for a "C" among "O" distractors. The search items were either holes or objects, defined by motion, contrast, or both. Our main findings were (1) it was easier to search among objects than to search among holes, and (2) the difference between search among objects and search among holes was primarily caused by grouping with the background. The data support the hypothesis that the shape of a hole is only available indirectly. We further note that, in our experiments, search performance for both holes and objects depended on the surface medium used to define the search items.
\end{abstract}

How do we perceive holes? Nelson and Palmer (2001) discussed which factors determine whether a surrounded region is perceived as a hole, or whether it is perceived as an object. They used a direct reporting task, where participants had to indicate on a scale from -100 to 100 whether they saw a hole or an object. They found that participants tended to detect a hole as a function of three factors: (1) the relative depth of the inner part of the hole area (e.g., based on shading); (2) the figural properties of the boundary surrounding the hole; and (3) the degree to which the inner part of the hole grouped with a "far" region, surrounding the local region, which itself immediately surrounds the hole. A hole was more likely to be seen if the inner part was further in depth than the local surround, if the boundary around the hole was concave and simple, and if the hole grouped with an outer, relative to an inner, surrounding region.

The question of whether and when a hole is perceived to have a shape of its own remains to be answered. Rock, Palmer, and Hume (cited in Palmer, 1999) found that recognition memory for holes was as good as for objects, even though the participants accurately perceived the holes as holes and the objects as objects on their initial presentation. According to Palmer, "the hole is ground for purposes of defining depth relations and what is material versus open space, but is figure for purposes of describing shapes" (p. 287). A similar suggestion is made by Peterson (2003).

A different proposal has been put forward by Bertamini and Croucher (2003), who examined the ease with which observers judged the relative heights of two vertices. Normally, such judgments are made more rapidly when the vertices are convex than when they are concave (Ber-

This work was supported by the U.K. Medical Research Council. Correspondence should be addressed to J. Hulleman, Department of Psychology, University of Hull, Hull HU6 7RX, England (e-mail: jhulleman@hull.ac.uk). tamini, 2001; Gibson, 1994). Bertamini and Croucher presented either a convex shape (an hourglass) or a concave shape (a barrel), either on a large background (the object condition) or with a local surround, with the color of the inside part then matching the wider background (the hole condition; see Figure 1). The participants' task was to judge which of the two vertices was lower. In the object condition, judgments were faster for the convex/barrel stimulus than for the concave/hourglass stimulus. However, this advantage was reversed when the local surround was present (now performance was better with the hourglass than with the barrel; in a follow-up study, Bertamini \& Mosca [2004] confirmed this finding using stereograms). Bertamini and Croucher interpreted their result as indicating that the contours were assigned to the local surround and not to the inner hole. With the contours assigned to the local surround, the surrounding object had convex contours around the hourglass hole, whereas it had concave contours around the barrel hole. Bertamini and Croucher suggested that "a hole is defined by the contour of the enclosing object, rather than the hole itself possessing the contour" (p. 52).

In the present study, we used visual search procedures to assess whether, in addition to any differences in representation when single stimuli are presented, holes contrast with objects in their ability to attract attention, even when holes and objects share the same contours. We report data from six experiments where, in each study, participants had to search for a target "C" among "O" distractors. When these stimuli are presented as static contours, this is usually an easy search task, little affected by the number of distractors present (Treisman \& Souther, 1985).

The novel element of our study was to surround each letter by a local square, with the local squares appearing on a larger background containing a random pixel array (see Figures 2 and 3 for examples). By varying the relations between the letter shape, its local surround, and the larger background area, we could present the letter stim- 


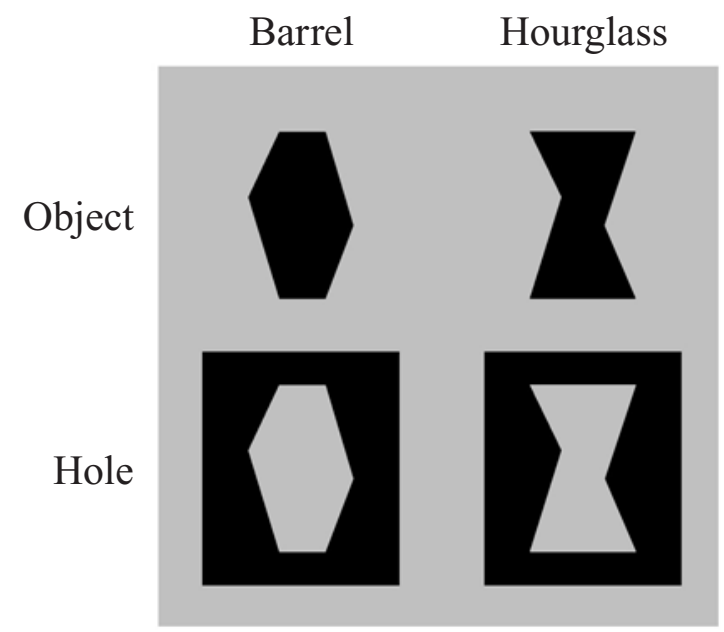

Figure 1. Items used in the experiments by Bertamini and Croucher (2003). The task of the participants was to judge which of the two vertices on the outside of the central shape was lower (vertically).

uli as either objects (e.g., the letter appeared as a figure against its local surround) or as holes (e.g., the letter grouped with the larger background, and the local surround appeared as the perceptual figure). Even if holes can be assigned a figural shape when focally attended (Rock et al., cited in Palmer, 1999), the more automatic process may be to initially assign the bounding edges of a hole to the figural surround.

Accordingly, under the multielement conditions used in search, any assignment of the bounding edges of holes to the local surround will make search slower than when the stimuli are coded as objects. Any contrast in the assignment of the bounding edges of the letter region to the target shape (object search) on the one hand, or to the surrounding region (search for stimuli defined as holes) on the other, would fit with the letter region being respectively coded either as figure (object search) or ground (search for holes, see Baylis \& Driver, 1995). A variation in figure-ground assignment may in turn affect performance in its own right (in addition to effects due to changes in contour assignment). Lamme and colleagues (Lamme, 1995; Lamme, Rodriguez-Rodriguez, \& Spekreijse, 1999) have shown that the activation of neurons in V1 depends on whether their receptive field contains a part of the stimulus that belongs to the ground or a part that belongs to the figure in a display.

In the present experiments, the relations among the letter stimulus, the local surround, and the larger background were varied using either random dot motion (Experiment 1), contrast (Experiment 2), or contrast and motion (Experiment 3). In these experiments, either the letter region (hole condition) or the local surround (object condition) had the same properties as the larger background. In Experiments 4-6, both the letter region and the local surround differed from the larger background. This allowed us to test whether grouping with the background (as identified by Nelson \& Palmer, 2001) contributes to the coding of a region as an object or a hole, thus differentially affecting performance for holes.

\section{GENERAL METHOD}

\section{Method}

Apparatus. The experiments were controlled by a Pentium III PC. The stimuli were presented on a 17-in. VGA monitor, in $800 \times$ 600 graphics mode.

Stimuli. The search stimuli in all the experiments were defined as either a "C" or an "O" (see Figures 2 and 3 ). These letters were presented as objects or as holes, according to the relations among the letters, their local surround, and their background. In Experiments 1-3, either the letter shapes or the local surround had surface

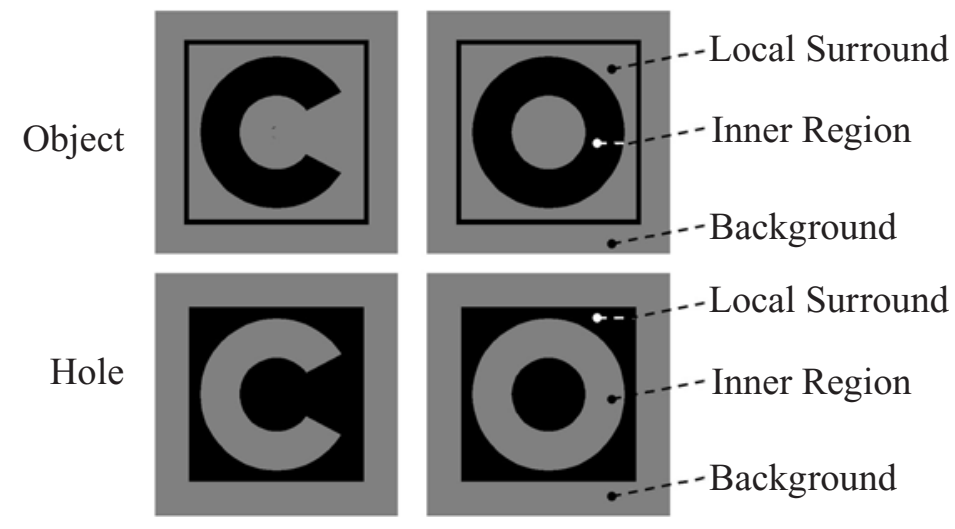

Figure 2. Items used in the experiments. In the object conditions of Experiments 1-3, the properties of the local surround and the background were identical and different from the inner region. In the hole conditions of Experiments 1-3, the properties of the inner region and the background were identical. Here, the local surround had different properties. In Experiments 4-6 for both the control object and control hole conditions, the properties of the inner region, the local surround, and the background were different from each other. 
Experiment 1

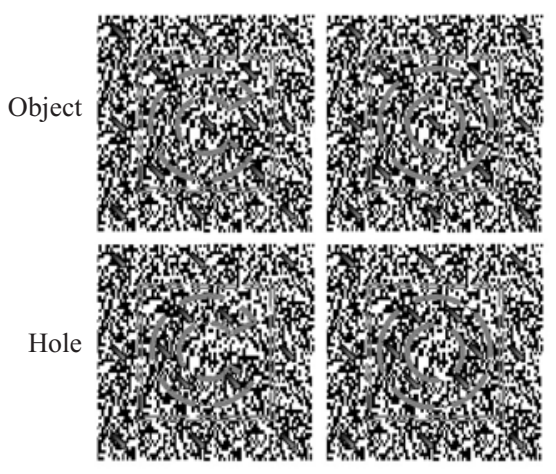

Experiment 4

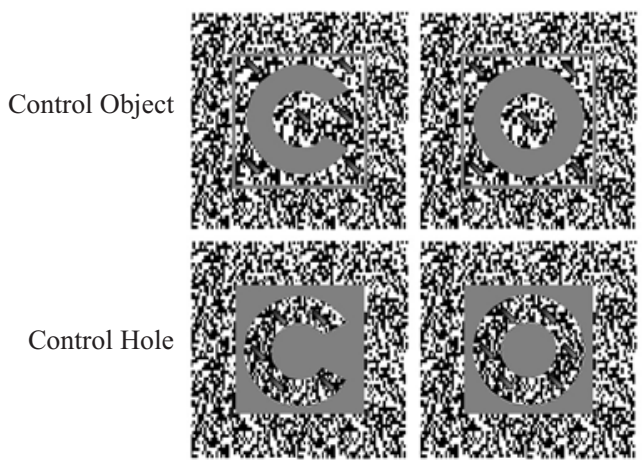

Experiment 2

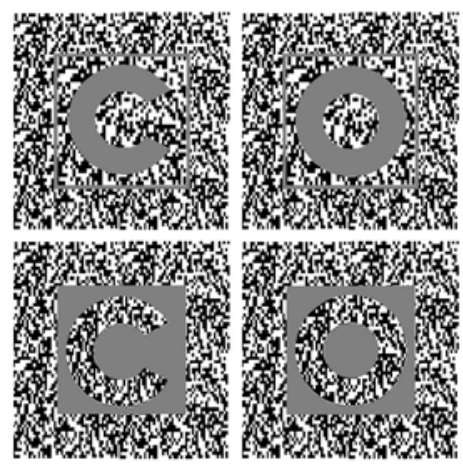

Experiment 5

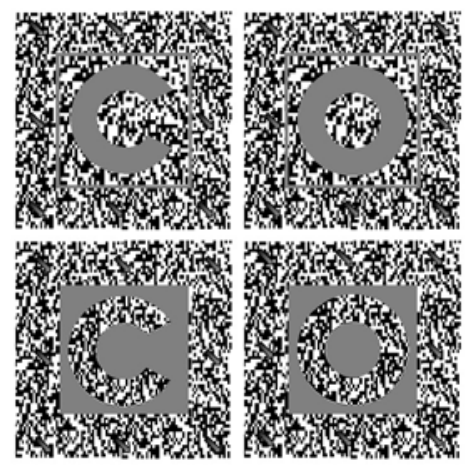

Experiment 3

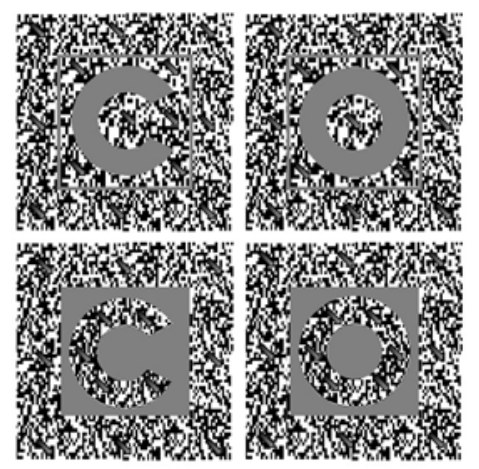

Experiment 6

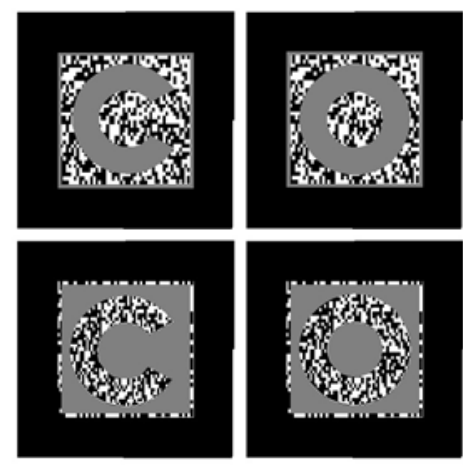

Figure 3. Overview of the experiments. Motion is indicated by the arrows. Experiment 1: Items defined by motion only. Experiment 2: Items defined by contrast only. Experiment 3: Items defined by both contrast and motion. Experiment 4: Motion inside the item, on a static background. Experiment 5: Static items on a moving background. Experiment 6: Static items on a black background.

properties (defined by motion, contrast, or contrast and motion) that were identical to the background. The other region differed from the background. In the object condition, the local surround had the same properties as the background. In the hole condition, the letter shape had the same properties as the background. In Experiments $4-6$, the surface properties of the letter shape, the local surround, and the background all differed from each other. Target and distractor letters then appeared as shapes against a local surround, which in turn was surrounded by a wider background in both the control object and in the control hole conditions. We have added the word control because the difference between the control object and control hole conditions in Experiments 4-6 is much more nominal than the difference between the object and hole conditions in Experiments 1-3. Using the control object and control hole conditions, we evaluated whether the slow search for the hole stimuli in Experiments 1-3 was due to grouping based on the surface properties of the letter stimulus and the wider background. If grouping contributes to the letter being coded as a hole, the difference between the control object and control hole conditions in Experiments 4-6 should be smaller than the difference between the object and hole conditions in Experiments 1-3.

With the exception of Experiment 6 (in which it was black), the background always consisted of a random pixel array. Depending on the experiment, the background was either static (Experiments 2, 4, and 6) or moving (Experiments 1, 3, and 5). In the experiments with a moving background, each frame was shifted one pixel both horizontally and vertically; therefore, the movement was always from top left to bottom right. The speed of the movement was about $1.9 \%$ sec. The regions of the display not matching this background (the letter, its local surround, or both) consisted either of a static random pixel array, or zero-contrast gray.
The letters and their local surrounds were presented on a virtual $10 \times 10$ grid, of which only the central 16 cells were used. The use of the central region alone was necessary to enable the participants to complete their task before the final frame was shown. Each letter plus its local surround subtended an area of $2.1^{\circ} \times 2.1^{\circ}$. An outer rim with a thickness of $0.1^{\circ}$ was included to match, as far as possible, the appearance of an object item with the square area around each hole item. The outer diameter of the "O" was $1.7^{\circ}$, and its inner diameter was $0.5^{\circ}$. To turn an "O" into a "C," a pie slice of $70^{\circ}$ was cut out of it. The grid cells were $3.5^{\circ} \times 2.6^{\circ}$.

Procedure. The participants were tested individually. There was an initial practice block of 16 trials and another practice block of 16 trials, if necessary. When the participants felt at ease with the task, they started the experimental blocks.

A trial consisted of a fixation cross presented in the center of the screen for $1,000 \mathrm{msec}$. After the fixation cross disappeared, a total of 76 frames were presented on the screen for $32 \mathrm{msec}$ each. The final frame remained on the screen until the participant responded or $15 \mathrm{sec}$ had passed. After the response, there was an intertrial interval of $1,000 \mathrm{msec}$, during which the frames for the next trial were generated.

The participants were asked to detect whether there was an item that was different from the others. We formulated the task in this way to prevent any biasing of the figure-ground interpretation of the search items. Nevertheless, after a few trials the participants likely realized the nature of the targets and distractors occurring in a trial; however, they defined the targets and distractors to themselves.

If a "C" was present, the participants had to push the "present" key on the keyboard as quickly as possible. If all the items were the same, they had to push the "absent" key. The present and absent keys were the " $\mathrm{Z}$ " and the "M," respectively, on a standard British keyboard. The assignment of the keys to either present or absent 
depended on the participant's preferred hand. Half pressed present with their preferred hand and half with their nonpreferred hand.

Design. All the experiments had the same design. They all contained the factors display size, stimulus type, and target. There were three levels for display size: 3,6 , or 9 items. There were two levels for both stimulus type (object, hole) and target (present, absent). The three factors were fully crossed. All 16 virtual cells in the display were occupied by a target item twice. The " $\mathrm{C}$ " item was always the target. This resulted in $3 \times 2 \times 2 \times 16 \times 2=384$ trials per experiment. Error trials were not retaken. Every experiment was subdivided into blocks of 96 trials. After each block, feedback was given on the error rate.

\section{EXPERIMENT 1 Form From Motion}

In Experiment 1, the search items were defined by form from motion. The entire search display consisted of black and white pixels. In the object condition, the local surround of the letter contained motion, as did the wide background. The pixels in the letter shape were static. In the hole condition, the inner region of the letter and the wide background moved; the local surround for each letter was static.

\section{Method}

Participants. Seventeen participants (14 female, 3 male) were recruited. All the participants had normal or corrected-to-normal vision, and they were naive about the purpose of this experiment.
Stimuli. Examples of the stimuli are shown in Figure 3. Both object and hole items were presented on a moving random pixel background. For the object items, the letter shape contained static pixels and the local surround contained moving pixels. For the hole items, the letter shape contained moving pixels and the local surround contained static pixels. The items were defined by motion only. This meant that, following the final frame, the search items receded into the background.

\section{Results}

The results are shown in Figure 4. The slopes are presented in Table 1. A three-way multivariate analysis of variance (MANOVA; stimulus type $\times$ display size $\times$ target) on the reaction times (RTs) yielded significant main effects of stimulus type $[F(1,16)=42.1, p<.001]$, display size $[F(2,15)=194.2, p<.001]$, and target $[F(1,16)=287.4, p<.001]$. There were also significant interactions between stimulus type and display size $[F(2,15)=15.8, p<.001]$ and between display size and target $[F(2,15)=121.8, p<.001]$.

A similar MANOVA on the error data revealed significant main effects: stimulus type $[F(1,16)=31.6, p<$ $.001]$, display size $[F(2,15)=30.6, p<.001]$, and target $[F(1,16)=112.7, p<.001]$. All two-way interactions were significant as well: between stimulus type and display size $[F(2,15)=5.5, p<.02]$, between stimulus type and target $[F(1,16)=16.1, p<.001]$, and between display size and target $[F(2,15)=31.4, p<.001]$. There was also
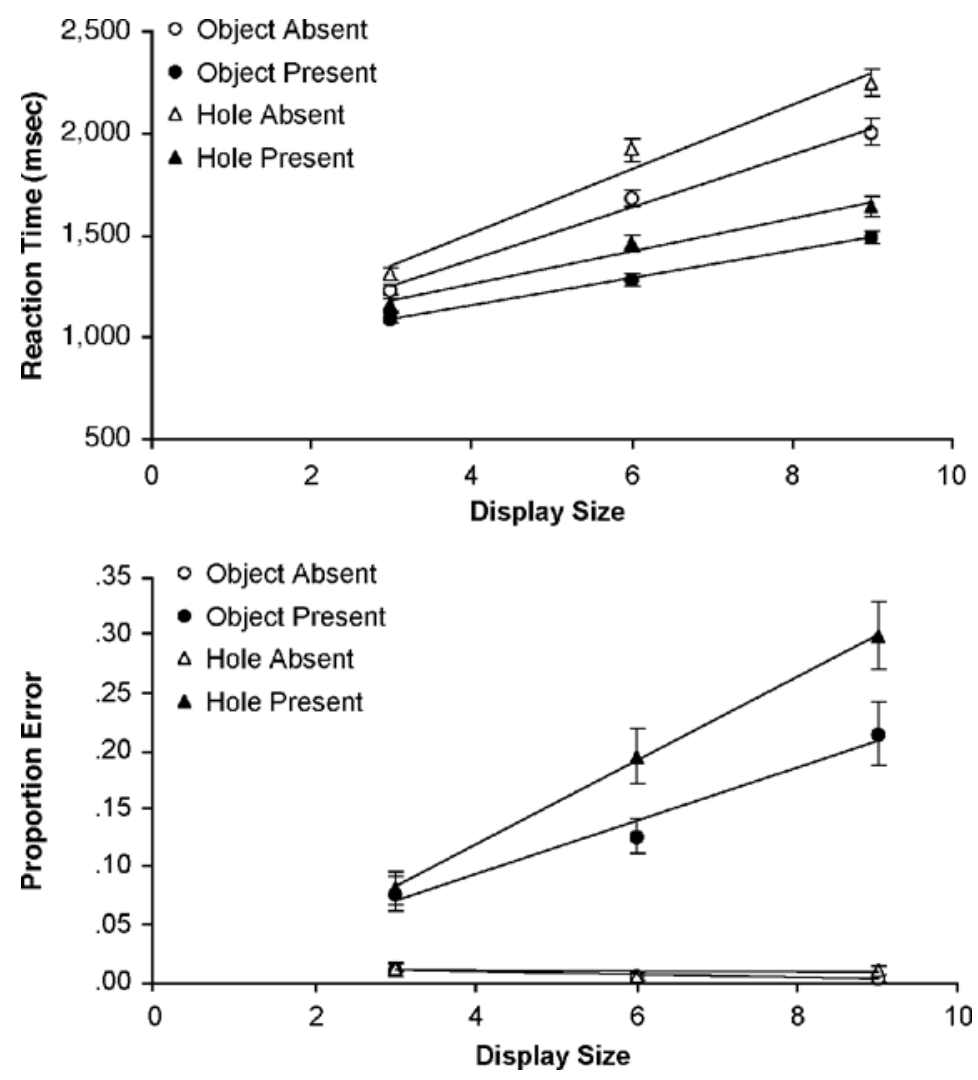

Figure 4. Results of Experiment 1: Items defined by form from motion. Error bars indicate standard errors of the means. 
Table 1

Slopes and Intercepts for Experiments 1-3

\begin{tabular}{|c|c|c|c|c|c|}
\hline Experiment & Defined by & Target & Item & $\begin{array}{c}\text { Slope } \\
\text { (msec/item) }\end{array}$ & $\begin{array}{c}\text { Intercept } \\
(\mathrm{msec})\end{array}$ \\
\hline \multirow[t]{4}{*}{1} & \multirow{4}{*}{ Motion } & \multirow{2}{*}{ Absent } & Object & 129 & 862 \\
\hline & & & Hole & 157 & 879 \\
\hline & & \multirow[t]{2}{*}{ Present } & Object & 67 & 884 \\
\hline & & & Hole & 80 & 935 \\
\hline \multirow[t]{4}{*}{2} & \multirow[t]{4}{*}{ Contrast } & \multirow[t]{2}{*}{ Absent } & Object & 36 & 676 \\
\hline & & & Hole & 106 & 718 \\
\hline & & \multirow[t]{2}{*}{ Present } & Object & 12 & 683 \\
\hline & & & Hole & 57 & 733 \\
\hline \multirow[t]{4}{*}{3} & \multirow[t]{4}{*}{ Contrast and motion } & \multirow[t]{2}{*}{ Absent } & Object & 37 & 615 \\
\hline & & & Hole & 88 & 732 \\
\hline & & \multirow[t]{2}{*}{ Present } & Object & 7 & 649 \\
\hline & & & Hole & 43 & 728 \\
\hline
\end{tabular}

a significant three-way interaction between stimulus type, display size, and target $[F(2,15)=3.9, p<.05]$.

There were more errors in the absent trials than in the present trials, both for the object and the hole items. This is a common result for difficult search tasks (Chun \& Wolfe, 1996), due to participants responding "absent" in the larger display sizes before completing their search on all the items. Nevertheless, the increase in error rate was much larger for the hole items, thereby supporting the conclusions from the RT analysis.

\section{Discussion}

There are two important points to note. The first is that search was quite difficult. The shallowest search slope was $67 \mathrm{msec} /$ item (object, present), and there were up to $30 \%$ errors in the hardest condition (hole, present). The difficulty of search even in the object condition is quite surprising given that previous investigations have indicated efficient search for targets defined by form from motion (Cavanagh, Arguin, \& Treisman, 1990), at least when the stimuli differ in the presence of a simple feature (as they do in this case; Treisman \& Souther, 1985). But there may be alternative explanations for the result we found. For example, the shapes of the items were more complex here than in the study by Cavanagh et al., and there may be some limitation on the efficiency of computing complex form from motion across a display.

The second point concerns the difference between searching among objects and searching among holes. Even though all the search slopes were rather steep, there still was a substantial advantage in terms of the slopes of the search functions for object relative to hole stimuli. This suggests that the shape characteristics that normally support search for a "C" among "O" become less available when both the " $\mathrm{C}$ " and the "O" are interpreted as holes. Nevertheless, the participants were able to perform the search task in the hole condition. So, even though the shape of the hole might be less readily available, it can nonetheless be coded, at least under attentional scrutiny. In this sense, our results are consistent with those of both Bertamini and Croucher (2003) and Rock et al. (cited in Palmer, 1999).
However, our conclusions about differences between holes and objects need to be guarded, since the movement of the random pixels in the letter region for the hole condition might have disrupted search. To overcome this, we differentiated the search items from the background by contrast only in Experiment 2. If the movement of the random pixels in the letter region causes the difference between the hole condition and the object condition in Experiment 1, we would expect this difference to disappear in Experiment 2. In Experiment 3, we differentiated the search items from the background by both contrast and motion. A comparison between Experiment 2 and Experiment 3 will enable us to gauge to what extent movement of the random pixel background contributes to the difference between the hole and object conditions.

\section{EXPERIMENT 2 \\ Form From Contrast and Luminance}

In Experiment 2, the items were defined by contrast (and luminance) differences relative to the background. Is the elimination of moving random pixels in the letter region enough to eradicate the difference between the hole and object stimuli?

\section{Method}

Participants. Sixteen new participants (13 female, 3 male) were recruited. All the participants had normal or corrected-to-normal vision, and they were naive about the purpose of this experiment.

Stimuli. Examples of the stimuli are shown in Figure 3. Both object and hole items were presented on a static random pixel background. For the object items, the letter shape contained uniform gray pixels, and the local surround consisted of static random pixels. For the hole items, the letter shape contained the random pixels, and the local surround contained the uniform gray pixels.

\section{Results}

The results are shown in Figure 5; the search slopes are listed in Table 1. A three-way MANOVA (stimulus type $\times$ display size $\times$ target) on the RTs yielded significant main effects of stimulus type $[F(1,15)=224.0$, $p<.001]$, display size $[F(2,14)=22.1, p<.001]$, and target $[F(1,15)=40.2, p<.001]$. There were also sig- 

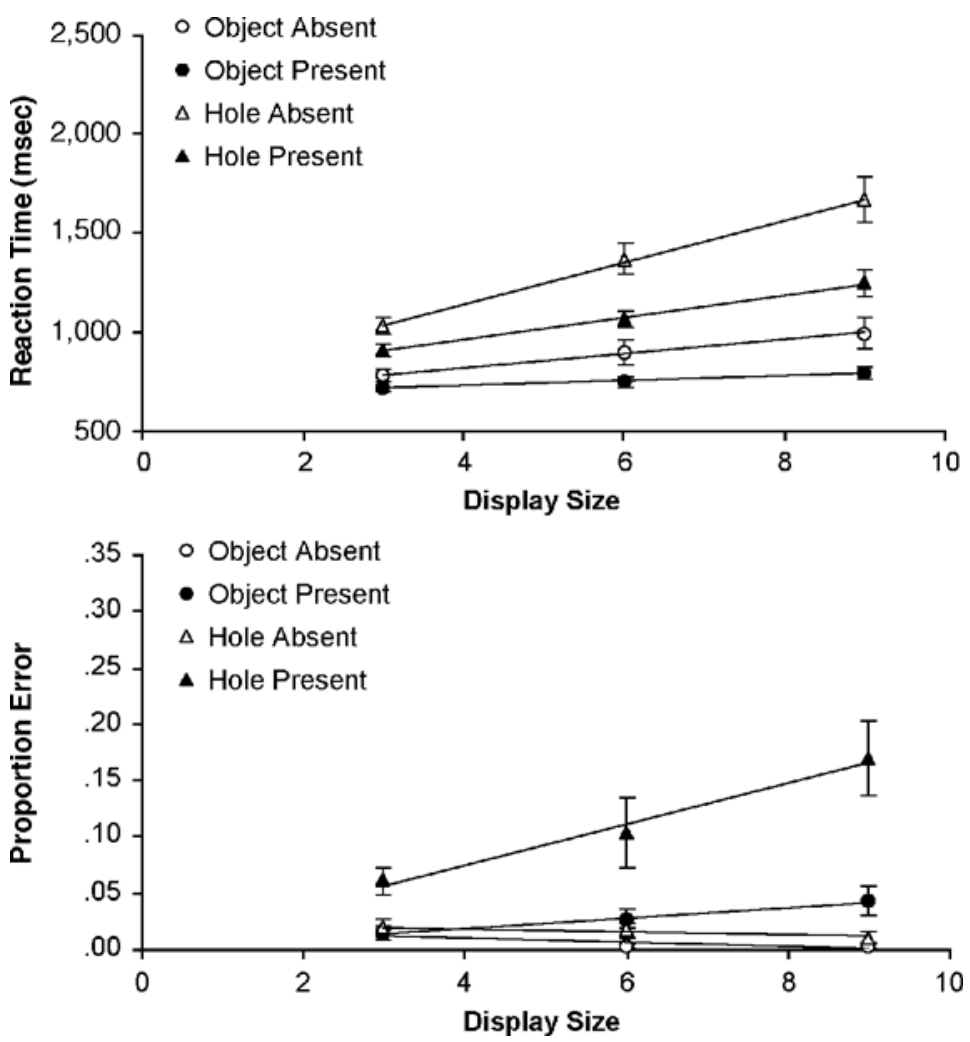

Figure 5. Results of Experiment 2: Items defined by contrast. Error bars indicate standard errors of the means.

nificant interactions between stimulus type and display size $[F(2,14)=24.4, p<.001]$, stimulus type and target $[F(1,15)=12.0, p<.004]$, and display size and target $[F(2,14)=18.0, p<.001]$.

A similar MANOVA on the error data revealed significant main effects: stimulus type $[F(1,15)=15.4, p<$ $.005]$, display size $[F(2,14)=5.5, p<.02]$, and target $[F(1,15)=26.5, p<.001]$. There were significant two-way interactions between stimulus type and target $[F(1,15)=9.5, p<.01]$ and between display size and target $[F(2,14)=13.3, p<.001]$. The three-way interaction between stimulus type, display size, and target was also reliable $[F(2,14)=5.4, p<.02]$. The interaction between stimulus type and display size was close to significance $[F(2,14)=3.4, p<.07]$.

\section{Discussion}

Search was much easier with the displays in Experiment 2 than with the displays in Experiment 1, with the slope of the search function on present trials for object targets being $12 \mathrm{msec} /$ item. This suggests that the surface medium defining the search items clearly had a large effect on performance (form from motion in Experiment 1; contrast in Experiment 2). We will return to this issue in the General Discussion section.
Despite search being much easier, clear differences between the hole and object conditions were still found. This indicates that movement of the random pixels in the letter region was not responsible for the difference between the hole and object conditions in Experiment 1. It seems, therefore, that the shape information derived from the hole stimuli does not distinguish very well between targets and distractors. This, in turn, suggests that the shape of the hole itself is not readily available to direct visual search, and the slope of the function for the hole stimuli was comfortably within the range usually taken to indicate serial search (Treisman \& Souther, 1985). The shape of the hole may only be discriminated through attentional scrutiny.

If anything, the slope difference between the hole and object conditions increased from Experiment 1 to Experiment 2 . This may have been caused by a floor effect in Experiment 1. There, the search items receded into the background after the last frame, effectively putting an upper limit on the RTs. However, it is also possible that the moving background in Experiment 1 interfered with search. In Experiment 3, we repeated Experiment 2 with search items defined by motion, as well as contrast. To what extent will a moving random pixel background reduce the difference between the hole and object conditions 

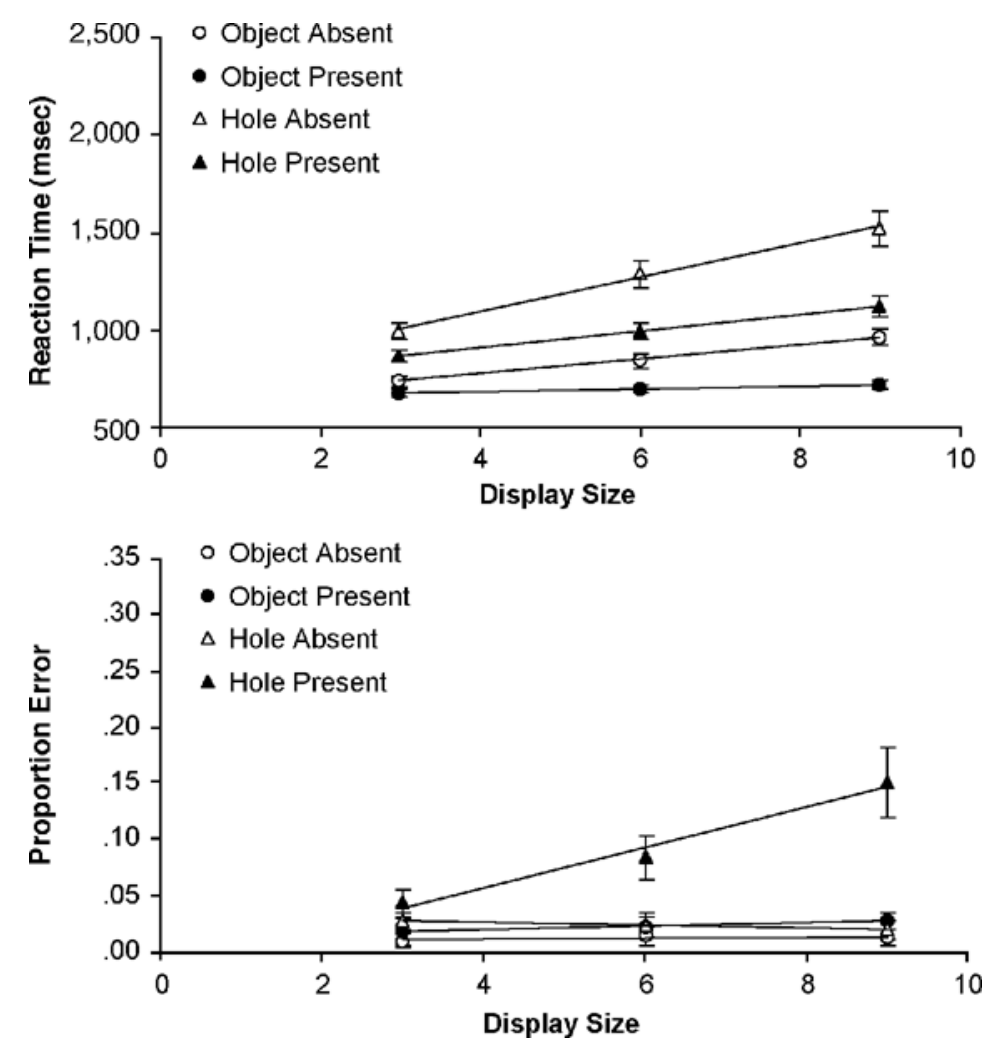

Figure 6. Results of Experiment 3: Items defined by motion and contrast. Error bars indicate standard errors of the means.

(by disrupting search), even when the letter shape (in the object condition) and the local surround (in the hole condition) are defined by contrast as well?

\section{EXPERIMENT 3 Form From Contrast and Motion}

In Experiment 3, we combined the characteristics defining the items in Experiments 1 and 2, so that both contrast and motion differentiated the search items from the background. Will the addition of moving random pixels change the difference between the hole and object conditions found in Experiment 2?

\section{Method \\ Participants. Sixteen new participants (all female) were re- cruited. All the participants had normal or corrected-to-normal vi- sion, and they were naive about the purpose of this experiment. \\ Stimuli. Examples of the stimuli are shown in Figure 3. Both ob- ject and hole items were presented on a moving random pixel back- ground. For the object items, the letter shape contained uniform gray pixels, and the local surround contained moving random pixels. For the hole items, the letter shape contained the moving random pixels, and the local surround consisted of uniform gray pixels.}

\section{Results}

Figure 6 shows the results. The search slopes are listed in Table 1 . A three-way MANOVA (stimulus type $\times$ display size $\times$ target) on the RTs yielded significant main effects of stimulus type $[F(1,15)=119.9, p<.001]$, display size $[F(2,14)=48.2, p<.001]$, and target $[F(1,15)=116.0, p<.001]$. There were also significant two-way interactions between stimulus type and display size $[F(2,14)=46.9, p<.001]$, stimulus type and target $[F(1,15)=25.3, p<.001]$, and display size and target $[F(2,14)=37.8, p<.001]$. Moreover, the three-way interaction stimulus type, display size, and target was significant $[F(2,14)=7.5, p<.007]$.

A similar MANOVA on the error data revealed significant main effects: stimulus type $[F(1,15)=40.1, p<$ $.001]$, display size $[F(2,14)=8.9, p<.005]$, and target $[F(1,15)=31.3, p<.001]$. All two-way interactions were significant as well: between stimulus type and display size $[F(2,14)=3.9, p<.05]$, stimulus type and target $[F(1,15)=16.5, p<.002]$, and display size and target $[F(2,14)=12.5, p<.001]$. The three-way interaction between stimulus type, display size, and target was also reliable $[F(2,14)=3.9, p<.05]$.

\section{Discussion}

The results of Experiment 3 closely match those found in Experiment 2 (and indeed, an across-experiment comparison of both RTs and error rates failed to demonstrate any differences between the studies; all $F \mathrm{~s}<1.13$ for the RTs and all $F \mathrm{~s}<2.00$ for the error rates). Search was again efficient for the object condition (slope $=7 \mathrm{msec} /$ item on present trials) but considerably slower for the hole condi- 

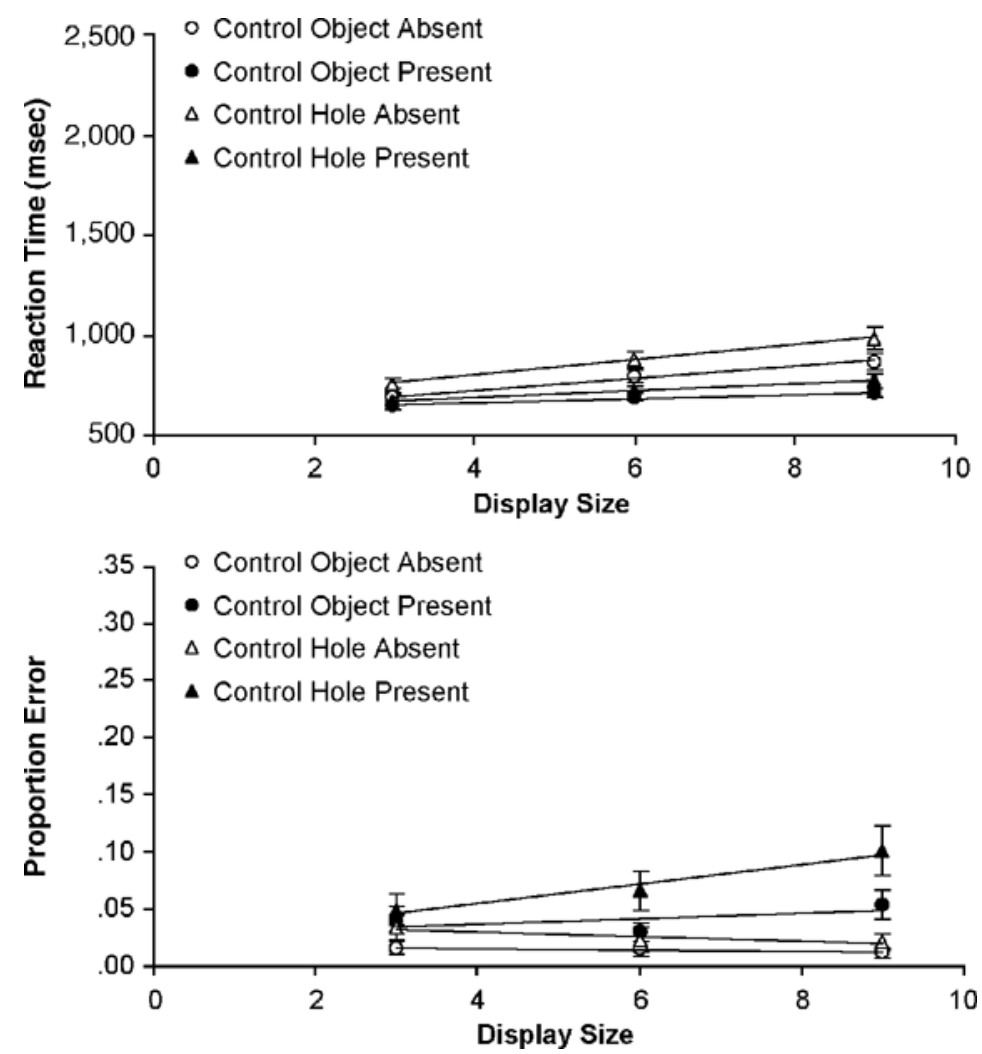

Figure 7. Results of Experiment 4: Items defined by contrast and motion of pixels on a static pixel background. Error bars indicate standard errors of the means.

tion. This shows that the movement of the random pixel background does not by itself have a large influence on the difference between the hole and object conditions. Also, as before, search for the hole stimuli was difficult (slope $=$ $43 \mathrm{msec} /$ item on present trials). This is again consistent with the view that the shape of the hole becomes available only under conditions of focused attention.

One factor that can contribute to the coding of a region as a hole is whether it groups with a larger background region (Nelson \& Palmer, 2001). In Experiments 4-6, we went on to test whether grouping between the letter regions and the wider background contributed to the advantage for objects over holes in search.

In Experiment 4, the background was a static random pixel array, whereas either the letter region of the search items contained a moving random pixel array and the local surround consisted of uniformly gray pixels (control hole), or these local relations were reversed (control object). In Experiment 5, we switched this assignment of the random pixel arrays: The letters and their local surrounds contained uniform gray and static random pixels, whereas the pixels in the wider background moved. In both experiments, the letter shapes in the control hole conditions shared their textures with the wider background but not its movement.

\section{EXPERIMENT 4 \\ Movement in the Search Item on a Static Wide Background}

\section{Method}

Participants. Sixteen new participants (13 female, 3 male) were recruited. All the participants had normal or corrected-to-normal vision, and they were naive about the purpose of this experiment.

Stimuli. Examples of the stimuli are shown in Figure 3. Both control object and control hole items were presented on a static random pixel background. For the control object items, the letter shape contained uniform gray pixels, and the local surround contained moving random pixels. For the control hole items, the letter shape contained moving random pixels, and the local surround consisted of static uniform gray pixels.

\section{Results}

The results are shown in Figure 7; the search slopes are presented in Table 2. A three-way MANOVA (stimulus type $\times$ display size $\times$ target) on the RTs yielded significant main effects of stimulus type $[F(1,15)=$ $52.7, p<.001]$, display size $[F(2,14)=36.7, p<.001]$, and target $[F(1,15)=43.0, p<.001]$. There were also significant two-way interactions between stimulus type and display size $[F(2,14)=8.5, p<.004]$, stimulus type and target $[F(1,15)=15.2, p<.002]$, and display size 
Table 2

\begin{tabular}{|c|c|c|c|c|c|}
\hline \multicolumn{6}{|c|}{ Slopes and Intercepts for Experiments 4-6 } \\
\hline \multirow{2}{*}{$\frac{\text { Experiment }}{4}$} & \multirow{3}{*}{$\begin{array}{l}\text { Defined by } \\
\text { pixel background, } \\
\text { on in mask }\end{array}$} & \multirow{3}{*}{$\frac{\text { Target }}{\text { Absent }}$} & Item & \multicolumn{2}{|c|}{$\begin{array}{c}\text { Slope Intercept } \\
(\mathrm{msec} / \text { item })(\mathrm{msec})\end{array}$} \\
\hline & & & Control object & 30 & 597 \\
\hline & & & Control hole & 38 & 620 \\
\hline & & Present & Control object & 10 & 640 \\
\hline & & & Control hole & 16 & 620 \\
\hline 5 & Static mask, moving pixel & Absent & Control object & 39 & 601 \\
\hline & background & & Control hole & 52 & 660 \\
\hline & & Present & Control object & 10 & 661 \\
\hline & & & Control hole & 23 & 637 \\
\hline 6 & Static mask, static black & Absent & Control object & 72 & 637 \\
\hline & background & & Control hole & 76 & 620 \\
\hline & & Present & Control object & 31 & 679 \\
\hline & & & Control hole & 26 & 660 \\
\hline
\end{tabular}

and target $[F(2,14)=13.8, p<.001]$. The three-way interaction between stimulus type, display size, and target was not significant.

In a similar MANOVA on the error rates, the following main effects were significant: stimulus type $[F(1,15)=$ $8.1, p<.02]$, display size $[F(2,14)=4.2, p<.04]$, and target $[F(1,15)=20.6, p<.001]$. The interactions between stimulus type and target $[F(1,15)=5.1, p<.04]$ and between display size and target $[F(2,14)=5.9, p<$ $.02]$ were significant as well.

\section{Discussion}

In this study, there was a small advantage for control object items over control hole items (slopes on present trials $10 \mathrm{msec} /$ item for control object stimuli and $16 \mathrm{msec} /$ item for control hole stimuli). In comparison with the earlier experiments, there clearly was a differential effect of decreased grouping between the background and the search items. Indeed, a statistical comparison between the object conditions of Experiments 2 and 3 and the control object condition of Experiment 4 (with display size and target as within-subjects factors and experiment as a between-subjects factor) did not yield a significant main effect of experiment nor any interaction involving experiment. A similar comparison between the hole conditions of Experiments 2 and 3 and the control hole condition of Experiment 4 however, yielded a significant main effect of experiment $[F(1,30)=39.4, p<$ .001 , and $F(1,30)=28.2, p<.001]$ for Experiments 2 and 3 , respectively. Moreover, there were interactions between experiment and display size $[F(2,29)=13.7, p<$ .001 , and $F(2,29)=14.2, p<.001]$, experiment and target $[F(1,30)=8.0, p<.01$, and $F(1,30)=12.3, p<.001]$, and between experiment, display size, and target $[F(1,30)=4.124, p<.03$, and $F(1,30)=5.5, p<.01]$ for the comparisons with Experiments 2 and 3, respectively.

When we compare the results in this experiment with those in Experiments 2 and 3, we do not find differences between the control object items and the object items. For the control hole items, however, performance was clearly improved by disrupting grouping. The search slopes were much steeper in Experiments 2 and 3 than in this experi- ment, which confirms that grouping with the overall background was important in generating the earlier differences between the hole and object conditions, presumably because grouping with the background prevented the letter region from being coded as a figure in the hole condition. Instead, we suggest that this area was probably assigned preattentively to the perceptual ground.

Even though grouping of the letter region and the wider background was disrupted, as attested by the search rates in the control hole condition, performance in the control object condition did not deteriorate in comparison with Experiments 2 and 3. In the object condition in the earlier experiments, grouping between the local surround and the wider background could have benefited performance (making the object stand out against its local surround). Hence, decreasing this grouping (in Experiment 4) might have decreased search efficiency, but there was no evidence for this. This, in turn, suggests that performance in all our experiments depends principally on grouping between the surface properties of the letter region and the larger background, rather than on grouping between the local surround around the letter and the larger background. Critically, in Experiments 1-3, the surface of the letter only groups with the wider background in the hole condition.

Experiment 5 examined the same issues, but had the background containing motion, whereas the random pixels in the search items were static.

\section{EXPERIMENT 5 Static Mask on a Moving Background}

\section{Method}

Participants. Sixteen new participants (11 female, 5 male) were recruited. All the participants had normal or corrected-to-normal vision, and they were naive about of the purpose of this experiment.

Stimuli. Examples of the stimuli are shown in Figure 3. Both control object and control hole items were presented on a moving random pixel background. For the control object items, the letter shape contained uniform gray pixels, and the local surround contained static random pixels. For the control hole items, the letter shape contained the static random pixels, and the local surround consisted of static uniform gray pixels. 


\section{Results}

The results are shown in Figure 8. The search slopes are listed in Table 2. A three-way MANOVA (stimulus type $\times$ display size $\times$ target) on RTs yielded significant main effects of stimulus type $[F(1,15)=72.3, p<.001]$, display size $[F(2,14)=15.9, p<.001]$, and target $[F(1,15)=23.8, p<.001]$. There were also significant two-way interactions between stimulus type and display size $[F(2,14)=15.0, p<.001]$, stimulus type and target $[F(1,15)=17.4, p<.001]$, and between display size and target $[F(2,14)=8.4, p<.005]$. The three-way interaction between stimulus type, display size, and target was not significant $[F(2,14)=1.4, p<.3]$.

In a similar MANOVA on error rates, only the main effect of mask type $[F(1,15)=8.5, p<.02]$ was significant. The interactions between mask type and display size $[F(2,14)=14.6, p<.001]$ and between display size and target $[F(2,14)=10.6, p<.002]$ were significant as well.

\section{Discussion}

In the control hole condition, the slopes were less efficient than in the equivalent condition in Experiment 4. A MANOVA on RTs with stimulus type, display size, and target as within-subjects factors and experiment as a between-subjects factor yielded a significant interaction between stimulus type and experiment $[F(1,30)=4.3$, $p<.05]$ and a significant interaction between stimulus type, display size, and experiment $[F(2,29)=4.6, p<$ $.02]$. All other effects involving experiment were not significant $(F \mathrm{~s}<1.4)$.

In addition, a comparison between the control hole condition here and the hole conditions of Experiments 2 and 3 (when the letter region grouped with the background) revealed a significant main effect of experiment $[F(1,30)=22.6, p<.001$, and $F(1,30)=13.9, p<.001]$ for Experiments 2 and 3, respectively. Moreover, there was an interaction between experiment and display size $[F(2,29)=6.9, p<.001$, and $F(2,29)=4.8, p<.02]$ for the comparisons with Experiments 2 and 3, respectively.

The interaction between display and experiment indicates that the slopes were steeper in Experiments 2 and 3 than they were in Experiment 5. The main effect of experiment indicates that RTs were slower in Experiments 2 and 3 .

A corresponding analysis of the object conditions from Experiments 2 and 3 and the control object condition from Experiment 5 failed to demonstrate any significant effects involving experiment (all $F \mathrm{~s}<1.2$ ). This
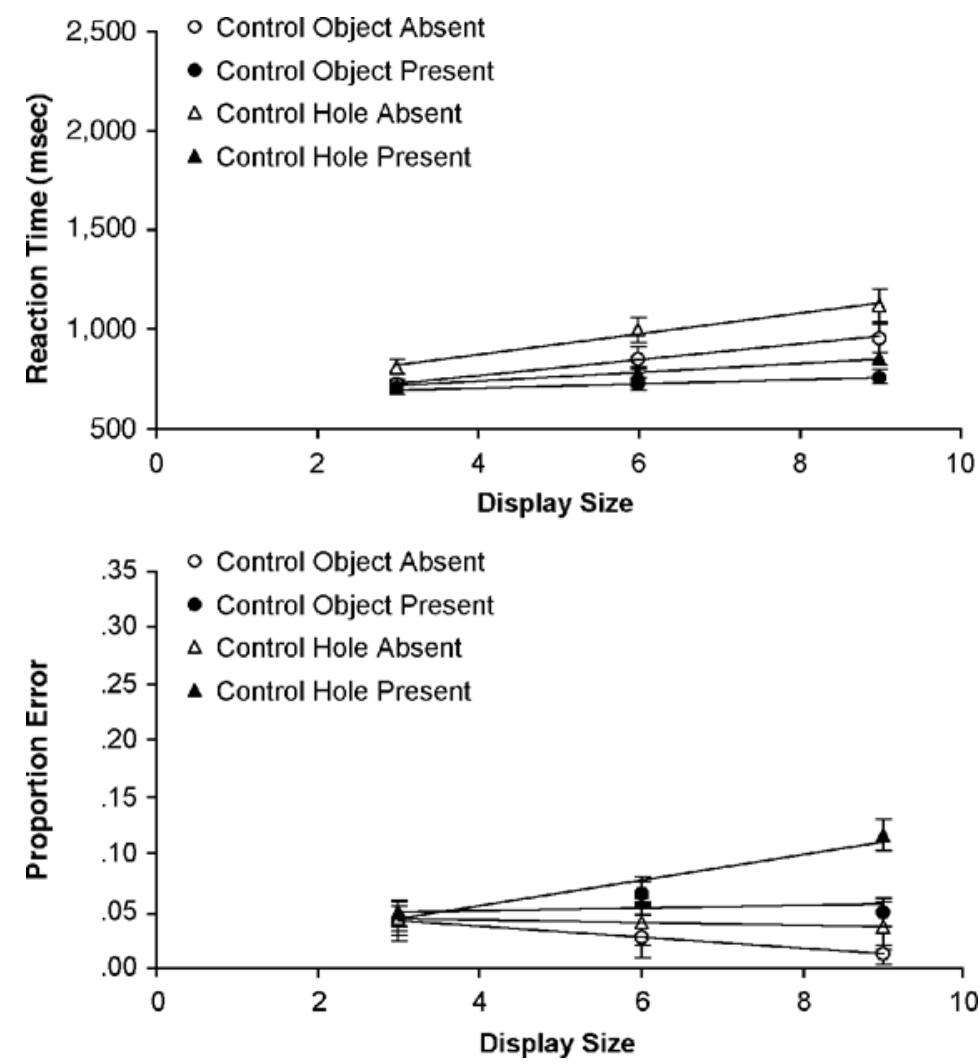

Figure 8. Results of Experiment 5: Items defined by contrast and static pixels on a moving pixel background. Error bars indicate standard errors of the means. 
confirms that there was a selective speeding of search in the control hole condition in comparison with the equivalent hole conditions in the earlier studies.

The pattern of results is similar to that observed in Experiment 4. Again we see that improved search due to disruption of grouping with background in the control hole condition is accompanied by an unchanged performance in the control object condition. In Experiment 5, the central letter region and its local surround were perceived to be in a plane in front of the moving background in both the control hole and control object conditions. Apparently, this did not impede search in the control object condition. Search in the control hole condition was less efficient than in the control hole condition of Experiment 4 but more efficient than in the hole conditions of Experiments 2 and 3. This might be an indication that the grouping between the central letter region and the background was disrupted less than in Experiment 4. Alternatively, there might have been some increased grouping between the central letter and the local surround. Both interpretations are consistent with the suggestion that predominantly grouping of the central letter region with the wider background is detrimental to search performance. Whenever this grouping is disrupted, search performance improves.

\section{EXPERIMENT 6 Static Items on a Static Black Background}

Experiment 6 was a final control study to assess whether the slowed search for the hole stimuli in Experiments 2 and 3 could be due to the letter being defined (at least in part) by a random array of black-and-white pixels. In the object items in these studies, the central letter was defined by uniformly gray pixels. Perhaps it is simply more difficult to search for a shape defined by a random pixel array than by uniformly gray pixels. This seems unlikely, given that the control hole displays in Experiment 4 used letters with random dot textures, too. Nevertheless, in Experiment 6 we used the same items as in Experiment 5 , but this time, we made the background uniformly black. If it is simply more difficult to perceive a shape defined by a random pixel array, we would again expect shallower slopes for control object items than for the control hole items.

\section{Method}

Participants. Ten new participants ( 8 female, 2 male) were recruited. All the participants had normal or corrected-to-normal vision, and they were naive about the purpose of this experiment.

Stimuli. Examples of the stimuli are shown in Figure 3. Both control object and control hole items were presented on a static
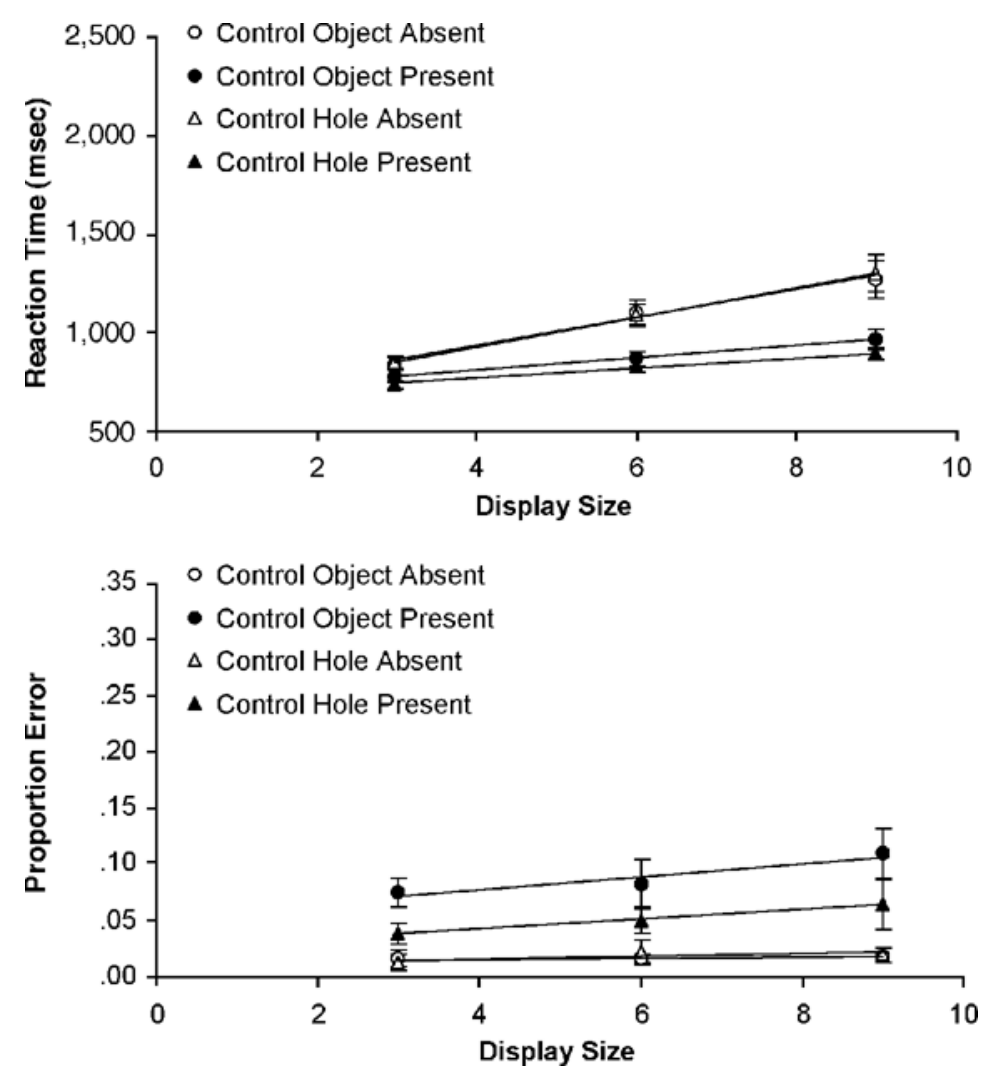

Figure 9. Results of Experiment 6: Items defined by contrast and static pixels on a black background. Error bars indicate standard errors of the means. 
black background. For the control object items, the letter shape contained uniform gray pixels, and the local surround contained static random pixels. For the control hole items, the letter shape contained static random pixels, and the local surround consisted of static uniform gray pixels.

\section{Results and Discussion}

The results are shown in Figure 9; the slopes are listed in Table 2. A three-way MANOVA (stimulus type $\times$ display size $\times$ target) on RTs yielded significant main effects of display size $[F(2,8)=21.9, p<.001]$ and target $[F(1,9)=40.6, p<.001]$. There were also significant two-way interactions between stimulus type and target $[F(1,9)=8.8, p<.02]$ and between display size and target $[F(2,8)=19.2, p<.001]$. The three-way interaction between mask type, display size, and target was not significant.

In a similar MANOVA on the error rates, the main effects of stimulus type $[F(1,9)=11.6, p<.008]$ and target $[F(1,9)=24.6, p<.001]$ were significant. The interaction between stimulus type and target $[F(1,9)=20.5$, $p<.002$ ] was significant as well.

If anything, in this study the control hole condition was actually easier than the control object condition. This emphasizes that the previously poor performance in the hole conditions cannot be attributed to the use of a random dot texture on the target letters per se.

A comparison between the object conditions of Experiments 2 and 3 and the control object condition of Experiment 6 (with display size and target as within-subjects factors and experiment as a between-subjects factor) reveals a significant interaction between display size and experiment $[F(2,23)=5.9, p<.01$, and $F(2,23)=10.7, p<$ .002 ] for Experiments 2 and 3, respectively. There was an increase in search slopes in the control object condition.

In Experiment 6, it became more difficult to search for the target shape in the control object condition. Experiment 6 itself does not distinguish between the possibility that performance in the control object condition deteriorated because the local surround grouped less with the background - making the letter region harder to distinguish from the local surround-or because the letter region itself grouped more with the background. However, in Experiment 5 (in the control hole condition) reducing the grouping of the central letter region with the background had a larger influence than did a potential increase in its grouping with the local surround. This would suggest that the letter region in the control object condition grouped more with the background in Experiment 6 . This would follow if areas with similar contrast grouped together during search. However, grouping with the background and grouping with the local surround might have been at work simultaneously, and further experiments will be necessary to establish their relative contributions. Both accounts underscore the influence of grouping, however. The relation between the central letter, its local surround, and the wider background determines the search rate rather than the texture of the letter shape itself.

\section{GENERAL DISCUSSION}

Two main results emerge from this paper. First, all else being equal, it was easier to search among objects than to search among holes. Second, this difference between search among objects and search among holes was strongly affected by grouping with the background. In addition, the speed of search in general depended on the surface medium (form from motion vs. form from contrast). We now consider each of these points.

\section{Search Among Objects Was Easier Than Search Among Holes}

The studies of Nelson and Palmer (2001) and Bertamini and Croucher (2003) have promoted recent interest in the perception of holes. Our visual search task offers another indirect way of investigating how holes are represented. In Experiments 1-3, participants performed much better in the object condition than in the hole condition, with large differences in slopes in both Experiments 2 and 3 . We suggest that the holes were slower to search through than the objects stimuli because with holes the bounding contour is initially assigned to the locally surrounding region rather than to the letter region itself. This means that the region of the hole is not immediately coded as a figure with its own shape. Its activation might therefore be reduced in comparison with the activation of the same stimulus when immediately coded as a figure (Lamme, 1995). Consequently, search may not be directed efficiently to a target defined by the shape of a hole. To discriminate such a target, we suggest that participants need to attend focally to each item in order to access the bounding contours of the letter region as a figural shape. This would account for how participants can discriminate a $\mathrm{C}$-shaped hole from an $\mathrm{O}$ shaped hole and why search for a C-shaped hole is less efficient. So the present effects reflect, at least in part, processes of figure-ground segmentation and hence implicit depth relations in the $2 \mathrm{D}$ displays. Such data fit with other examples where depth relations are coded sufficiently quickly to influence search slopes.

For instance, He and Nakayama (1992) showed that is very difficult to search for an L-target among mirrorreversed L-distractors when they can be amodaly completed into squares. Similarly, Rensink and Enns (1998) reported that search for the combination of a notched square and a circle among complete squares and circles was easy when the notched square was free from the circle but much more difficult when the notched square made contact with the circle (i.e., as if the circle were occluding a complete square). It appears that search operates on visual representations coded for their figureground relations in depths.

Substituting an object with a hole need not always be detrimental to performance, though. For instance, in one of the experiments conducted by Bertamini and Croucher (2003), there was actually an increase in performance when the stimulus was a hole rather than an object. In 
the hole stimulus, the critical aspect of the stimuli used by Bertamini and Croucher-a convex vertex-was more readily available.

\section{Grouping With the Background Was the Main Cause of the Difference Between Objects and Holes}

There are probably two defining features of a hole: (1) one is able to look through it, and (2) it is bounded by a single object. Each feature may have to be established before something qualifies as a hole. When other objects can be seen through a bounded region (e.g., the trees outside through an open window), this establishes its status as a hole. Another way of establishing that a bounded region is a hole, however, is the fact that the texture of the background, which extends beyond the object that contains the hole, is the same as the texture in the hole itself (cf. Nelson \& Palmer, 2001). On this view, grouping with the background is not the feature that defines a hole (that is, what we would call seethroughness and boundedness), but it is one of the ways of establishing that a bounded region is in fact a hole, rather than a textured object. The important role played by grouping with the background was illustrated in Experiments 4-6.

In Experiments 4 and 5, the effect of grouping with the background was reduced. This was done by giving the letter regions and their local surrounds characteristics that were no longer identical to the larger background. As a result, the slopes in the control hole conditions became shallower, even though performance in the control object conditions remained constant. This suggests that, in our search experiments, grouping of the central letter region with the background plays the most important role. Grouping of the central letter region with the background reduces search performance, whereas, in the absence of grouping with the background, the amount of grouping between the central letter region and the local surround (or between the local surround and the background) only seems to have limited influence.

Another illustration of the influence of grouping with the background is provided by a comparison between the control hole conditions of Experiments 4 and 5 and the hole conditions of Experiments 2 and 3. The search slopes varied greatly across these studies, though the target stimuli were the same. The slopes were much steeper when the background was identical to the central letter region than when these regions differed in their motion properties.

Experiment 6 changed the amount of grouping between the background, the central letter regions, and the local surrounds. As a result, the difference between the control hole and control object conditions disappeared, and there were steeper search slopes in the control object condition. Presumably, this was the result of an increase in grouping between the letter region and the wider background, although a decrease in grouping between the local surround and the background (reducing the distinction between the letter region and the local surround) may have had an influence as well. Further research will be needed to establish the factors that determine the strength with which regions will group, and the precise role of grouping between the local surround and the background.

\section{Search Performance Depended on the Surface Medium}

Cavanagh et al. (1990) reported that search items defined by form from motion yielded search slopes that were indistinguishable from the slopes for shapes that were defined by luminance. They used relatively simple visual search tasks with line elements, where the target was distinguished from the distractors by orientation. In contrast to this, we found that search for a "C" among "O"s was much more difficult when the target was defined by form from motion (Experiment 1) than when it was defined by contrast (Experiments 2 and 3).

What could account for the difference between the studies? One possibility is that form from motion can only yield fairly simple shape information in an efficient fashion across the field. With more complex shape discriminations, some degree of attention may need to be paid to each stimulus to help code shape detail. In the present case, discrimination of the "C" target from "O" distractors may simply be beyond the capacity of the form-from-motion system (though this same discrimination can be performed efficiently by a luminance-based system; Experiments 2 and 3 here; see also Treisman \& Souther, 1985).

Searching for an oblique rectangle defined by form from motion and searching for an oblique line of discontinuity in the motion of the background could constitute equally successful strategies in the experiments reported by Cavanagh et al. (1990). In their experiment, the surfaces were static, textured rectangles, presented on a moving, similarly textured background. To find the oblique target rectangle in an array of vertical distractor rectangles, it is sufficient to know the orientation of a single side of the rectangles. If the orientation was horizontal or vertical, the side belonged to a distractor rectangle. If the orientation was oblique, it was the target.

However, in our search task, a simple strategy based on detection of a diagnostic feature was not possible because both target and distractors were based on the same circle. Moreover, the horizontal lines in the " $\mathrm{C}$ " could not be used, because horizontal lines were also provided by either the surrounding square of the object items or the square local surround of the hole items. Our results suggest that form from motion may not be derived efficiently to serve at least more complex shape discriminations. However, the issue of surface medium was not the main topic of the present study. What is needed is a more systematic evaluation of the shape discriminations that can be performed across a display based on form from motion.

\section{Alternative Accounts}

Our results also allow us to discount two alternative explanations. The first of these is that the difference in performance for search among holes and search among objects is caused by particular characteristics of the hole 
masks. For instance, they might be more complex, or they might be more similar to each other because the central letter is always surrounded by a gray square. However, a comparison between the hole conditions of Experiments 2 and 3 on the one hand and the control hole conditions of Experiments 4 and 5, on the other, showed that the similarity between the inner region and the background determined the steepness of the search slopes, rather than the similarity or complexity of the hole masks per se. The search slopes for the holes were much steeper in Experiments 2 and 3 (about $50 \mathrm{msec} / \mathrm{item}$ ) than in Experiments 4 and 5 (about $20 \mathrm{msec} /$ item), despite the presence of the surrounding square mask in both cases.

A second alternative explanation is that the hole condition is hard because the letter regions were defined by a static or moving random pixel array rather than by a uniformly gray area. However, Experiments 3 and 4 showed that performance was not necessarily disrupted by the presence of a moving random pixel array in the letter region. Moreover, in Experiment 6, performance tended to be faster with the random pixel array letters than with letters defined by a uniformly gray region.

\section{CONCLUSION}

From our experiments, the following picture of visual search arises. To perform search, the visual system initially needs to establish the location of the search items. This process is affected by preattentive grouping between local areas and larger areas of background with similar properties. This grouping process occurs irrespective of whether it actually facilitates or impedes the search task. Areas that group with the overall background are coded as local regions of ground, leading to their interpretation as holes. Such regions do not guide search efficiently on the basis of the shape of their bounding contour. Search is guided more efficiently to the shape of regions (objects) that do not group with the wider background. In this sense, visual search is more concerned with objects than with the empty spaces surrounding them and surrounded by them.

\section{REFERENCES}

BAYLIS, G. C., \& Driver, J. (1995). Obligatory edge assignment in vision: The role of figure and part segmentation in symmetry detection. Journal of Experimental Psychology: Human Perception \& Performance, 21, 1323-1342.

Bertamini, M. (2001). The importance of being convex: An advantage for convexity when judging position. Perception, 30, 1295-1310.

Bertamini, M., \& Croucher, C. J. (2003). The shape of holes. Cognition, 87, 33-54.

Bertamini, M., \& Mosca, F. (2004). Early computation of contour curvature and part structure: Evidence from holes. Perception, 33, 35-48.

Cavanagh, P., Arguin, M., \& Treisman, A. (1990). Effect of surface medium on visual search for orientation and size features. Journal of Experimental Psychology: Human Perception \& Performance, 16, 479-491.

Chun, M. M., \& Wolfe, J. M. (1996). Just say no: How are visual searches terminated when there is no target present? Cognitive Psychology, 30, 39-78.

Gibson, B. S. (1994). Visual attention and objects: One versus two or convex versus concave? Journal of Experimental Psychology: Human Perception \& Performance, 20, 203-207.

He, Z. J., \& NaKayama, K. (1992). Surfaces versus features in visual search. Nature, 359, 231-233.

LAMmE, V. A. F. (1995). The neurophysiology of figure-ground segregation in primary visual cortex. Journal of Neuroscience, 15, 16051615.

Lamme, V. A. F., Rodriguez-Rodriguez, V., \& Spekreijse, H. (1999). Separate processing dynamics for texture elements, boundaries and surfaces in primary visual cortex of the macaque monkey. Cerebral Cortex, 9, 406-413.

Nelson, R., \& Palmer, S. E. (2001). Of holes and wholes: The perception of surrounded regions. Perception, 30, 1213-1226.

Palmer, S. E. (1999). Vision science: Photons to phenomenology. Cambridge, MA: MIT Press.

Peterson, M. A. (2003). On figures, grounds, and varieties of surface completion. In R. Kimchi, M. Behrmann, \& C. Olson (Eds.), Perceptual organization in vision: Behavioral and neural perspectives (pp. 87-116). Mahwah, NJ: Erlbaum.

RENSINK, R. A., \& EnNS, J. T. (1998). Early completion of occluded objects. Vision Research, 38, 2489-2505.

Treisman, A., \& Souther, J. (1985). Search asymmetry: A diagnostic for preattentive processing of separable features. Journal of Experimental Psychology: General, 114, 285-310.

(Manuscript received June 20, 2003; revision accepted for publication June 25, 2004.) 